\title{
Notes on Transliteration and Formatting
}

To maintain authenticity, I chose to follow the Hungarian spelling 1 convention. For example, Tokay wine was spelled Tokaji wine, street names were capitalized as "Andrássy út" rather than Andrássy Út, and last names were written ahead of first names, e.g., Kenéz Tibi rather then Tibi Kenéz. When necessary, the correct pronunciation was indicated in the text.

I italicized all quoted conversations to indicate that they were reconstructed from memory or assumed to have happened based on my knowledge of some or all of the participants. 